A divergent series which remains divergent whatever be the arrangement of its terms will be called unconditionally divergent.

Theorem III. - Every unconditionally divergent series tends towards infinity.

In fact, in such a series $g=1$, but this is impossible in a divergent series unless $N_{1}= \pm \infty$.

It is easy to see that conversely, every series which tends towards infinity is unconditionally divergent.

A divergent series which becomes convergent after a proper arrangement of its terms will be called conditionally divergent.

Theorem IV. - Every divergent series which does not tend towards infinity is conditionally divergent, and conversely, a conditionally divergent series cannot tend towards infinity.

Theorem V. - Every semi-convergent series can by a proper arrangement of its terms be made conditionally divergent, and conversely, every conditionally divergent series can by a proper arrangement of its terms be made semi-convergent.

Remark. - Riemann has proved that by a proper arrangement (commutative, not associative) of the terms of a semi-convergent series this series can be made to converge to any arbitrarily assigned number. It follows from the above that by a proper arrangement (both commutative and associative) of the terms of a conditionally divergent series this series can be made to converge to any arbitrarily assigned number.

The Johns Hopkins University, Baltimore, October, 1895.

\title{
A SIMPLE PROOF OF A FUNDAMENTAL THEOREM OF SUBSTITUTION GROUPS, AND SEVERAL APPLICATIONS OF THE THEOREM.
}

BY DR. G. A. MILLER.

Theorem. - The average number of elements in all the substitutions of a group is $n-\alpha, n$ being the degree of the group, and $\alpha$ the number of its transitive constituents.*

We shall first prove the theorem for $\alpha=1$, i.e. for the transitive groups.

* Frobenius, Crelle, vol. 101, p. 287. 
Let $g$ represent the order of such a group, and $a_{1}, a_{2}, \ldots, a_{n}$ its elements. Since the group contains only $g / n$ substitutions* that do not involve $a_{a}$ for each particular value of $\alpha$ in the series $\alpha=1,2, \cdots, n$, it must contain $g-g / n=\frac{n-1}{n} g$ substitutions that contain any one of the $n$ given $\alpha_{\alpha}$ 's. Hence all the substitutions of the group contain $\frac{n-1}{n} g n=(n-1) g$ elements. As the number of substitutions is $g$, the average number of elements in all of them must be $n-1$.

To see that the theorem is also true when $\alpha>1$, i.e. when the group is intransitive, it is only necessary to observe that every intransitive group may be resolved into transitive constituent groups, and that the substitutions of these transitive constituents are equally distributed among the substitutions of the intransitive group. $\dagger$

\section{Applications.}

For every substitution of a transitive group which contains less than $n-1$ elements there must be a sufficient number of substitutions of the $n$th degree to make the average number of elements in all these substitutions $n-1$. In particular, for identity there must be $n-1$ substitutions of the $n$th degree, since $\frac{0+(n-1) n}{n}=n-1$. Hence the formula $\ddagger$

$$
n_{n}=n-1+(n-3) n_{2}+(n-4) n_{3}+\cdots+n_{n-2},
$$

$n_{a}$ representing the number of substitutions in the group, which contain exactly a letters.

This formula is equivalent to the following: $\S$

$$
n_{n}=n\left(\frac{1}{2} n_{n-2}^{\prime}+\cdots+\frac{n-\alpha-1}{n-\alpha} n_{\alpha}^{\prime}+\cdots+\frac{n-1}{n} n_{0}^{\prime}\right) .
$$

Here $n_{a}^{\prime}$ bears the same relation to the subgroup which contains all the substitutions that replace a given letter by itself as $n_{a}$ bears to the entire group.

Let $H$ be the subgroup which is generated by all the substitutions of the $n$th degree in any transitive group $G$. If $H$ were intransitive, the average number of elements in all its substitutions would, according to the given theorem, be less

* Netro's Theory of Substitutions (CoLE's translation), § 61 .

$\dagger$ Bolza, American Journal, vol. 11, p. 200.

$\ddagger$ Cauchr, Comptes Rendus, vol. 21 (2), p. 897.

$\S$ Jordan, Comptes Rendus, vol. 74, p. 977. 
than $n-1$, while the average number of elements in all the substitutions of $G$ is $n-1$. This is impossible, as the substitutions of $G$ which are not also in $H$ cannot contain more than $n-1$ elements. Hence $H$ is transitive. Since the average number of elements in the substitutions of $G$ is the same as that in the substitutions of $H$, the substitutions of $G$ which are not found in $H$ must all be of the $(n-1)$ th degree.

The theorems of $\S \S 75,76$ of Netto's work * are proved by the preceding paragraph. It may be well to add in regard to the given $\S 75$ that $G$ may be transitive while the corresponding subgroup of $G$ is intransitive. The following group $\dagger$ is an instance :

\begin{tabular}{ll|ll}
1 & 1 & $A B \cdot C E$ & $a f . b j . c i . d h . e g$ \\
$A B C D E$ & abcde.fghij & $A C \cdot D E$ & $a g . b f . c j . d i . e h$ \\
$A C E B D$ & acebd.fhjgi & $A D \cdot B C$ ah.bg.cf.dj.ei \\
$A D B E C$ & adbec.figjh & $A E \cdot B D$ ai.bh.cg.df.ej \\
$A E D C B$ & aedcb.fjihg & $B E . C D, a j \cdot b i . c h . d g . e f$
\end{tabular}

Netto's statement: "If $G$ is transitive in the $A$ 's, $H$ is transitive in the $x$ 's," together with the rest of the section seems to me to imply that such a case is impossible.

LeIPZIG, August, 1895.

\section{ON AN UNDEMONSTRATED THEOREM OF THE DISQUISITIONES ARITHMETIC $Æ$.}

BY DR. JAMES PIERPONT.

The last section of the Disquisitiones Arithmeticæ contains the algebraic solution of the equations upon which the prime roots of unity depend.

$$
x^{p}-1=0, *
$$

where $p$ is a prime. As this equation contains the factor $(x-1)$, we may consider instead the equation

$$
x^{p-1}+x^{p-2}+\cdots+x+1=0 .
$$

\footnotetext{
* Cole's translation, pp. 86, 87.

$\dagger$ Cole, Quarterly Journal, vol. 27, p. 41.

$\ddagger$ The algebraic solution of these equations so simple in form presented difficulties which the mathematicians of the last century were not able to surmount. When $p=11$ one arrives at an equation of 5 th degree. Vandermonde gave the solution of this equation at the close of his paper Mémoire sur la Résolution des Equations, Hist. Acad. de Paris, 1771, but it appears to have been little known.
} 